\title{
Bis-indolic compounds as potential new therapeutic alternatives for tularaemia
}

\author{
Yvan Caspar $^{1,2 *}$, Vivien Sutera ${ }^{1,2}$, Sandrine Boisset ${ }^{1,2}$, Jean-Noël Denis ${ }^{3}$ and Max Maurin ${ }^{1,2}$ \\ ${ }^{1}$ Laboratoire de Bactériologie, Centre Hospitalier Universitaire de Grenoble, Université Joseph Fourier Grenoble-1, Grenoble, France \\ 2 Laboratoire Adaptation et Pathogénie des Micro-organismes, UMR-5163 CNRS, Université Joseph Fourier Grenoble-1, Grenoble, France \\ ${ }^{3}$ Département de Chimie Moléculaire (SeRCO), UMR-5250 CNRS, Université Joseph Fourier Grenoble-1, Grenoble, France
}

Edited by:

Anders Sjostedt, Umeå University,

Sweden

\section{Reviewed by:}

Lee-Ann H. Allen, University of

lowa, USA

Joseph Horzempa, West Liberty

University, USA

\section{*Correspondence:}

Yvan Caspar, Laboratoire

Adaptation et Pathogénie des

Microorganismes, Institut Jean

Roget, UMR-5163 CNRS, Université

Joseph Fourier Grenoble-1,

Domaine de la Merci, BP 170,

38042 Grenoble Cedex 9, France

e-mail: ycaspar@chu-grenoble.fr
Francisella tularensis is the etiological agent of tularaemia and a CDC class A biological threat agent. Few antibiotic classes are currently useful in treating tularaemia, including the aminoglycosides gentamicin and streptomycin, fluoroquinolones, and tetracyclines. However, treatment failures and relapses remain frequent and $F$ tularensis strains resistant to antibiotics have been easily selected in vitro. In this study, we evaluated the activity of new synthetic bis-indole derivatives against this pathogen. Minimum inhibitory concentrations (MICs) of four compounds (dcm01 to dcm04) were determined for the reference strains F. tularensis subsp. holarctica LVS NCTC10857, F. tularensis subsp. novicida CIP56.12 and F. philomiragia ATCC25015, and for 41 clinical strains of $F$. tularensis subsp. holarctica isolated in France. Minimal bactericidal concentrations (MBCs) were determined for the dcm02 and dcm04 compounds for the LVS and two clinical strains. Killing curves were also determined for the same three strains exposed to dcm04. All tested bis-indole compounds were bacteriostatic against $F$. tularensis subsp. holarctica strains, with a $\mathrm{MIC}_{90}$ of $8 \mu \mathrm{g} / \mathrm{mL}$ for dcm01, dcm02, and dcm03, and $2 \mu \mathrm{g} / \mathrm{mL}$ for dcm04. Only one strain was resistant to both $\mathrm{dcm} 01$ and dcm03, with $\mathrm{MICs}>32 \mu \mathrm{g} / \mathrm{mL}$. In contrast, F. tularensis subsp. novicida was resistant to all derivatives and F. philomiragia was only susceptible to dcm02 and dcm04, with MICs of 16 and $4 \mu \mathrm{g} / \mathrm{mL}$, respectively. $\mathrm{MBC}$ and killing curve experiments revealed significant bactericidal activity (i.e., 3-log reduction of the bacterial inoculum) of the dcm02 and dcm04 compounds only for the LVS strain. In conclusion, we have identified novel synthetic bis-indole compounds that are active against $F$. tularensis subsp. holarctica. They may be drug candidates for the development of new therapeutic alternatives for tularaemia treatment. Their further characterization is needed, especially identification of their bacterial targets.

Keywords: tularaemia, Francisella tularensis, bis-indolic compounds, antibacterial activity

\section{INTRODUCTION}

Francisella tularensis, the agent of the zoonosis tularaemia, may cause severe to fatal human infections. This intracellular, Gramnegative bacterium is highly infectious for humans and many animal species. No human-to-human transmission has been described so far and human infection may occur through direct contact with infected animals, ingestion of contaminated meat or water, arthropod bites, contact with contaminated environments and laboratory exposure to F. tularensis cultures (Dennis et al., 2001; Maurin et al., 2011). F. tularensis is a class A biological threat agent according to the CDC (Centers for Diseases Control and Prevention, Atlanta, Georgia, USA). The highly virulent $F$. tularensis subsp. tularensis strains (Jellison type A) are located in North America, whereas F. tularensis subsp. holarctica strains (Jellison type B) are found throughout the northern hemisphere. In Europe, tularaemia cases are often sporadic (Maurin et al., 2011), but outbreaks have recently been reported in many countries, including in Spain, Norway and Sweden (Pérez-Castrillón et al., 2001; Larssen et al., 2011; Rydén et al., 2012). The first-line therapy of tularaemia is based on a reduced number of antibiotics, including the aminoglycosides (gentamicin and streptomycin), the tetracyclines (e.g., doxycycline), and the fluoroquinolones (e.g., ciprofloxacin) (Johansson et al., 2002). Treatment duration is usually 7-10 days for gentamicin and ciprofloxacin, and 2-3 weeks for doxycycline. However, high rates of failure and relapse are observed in tularaemia patients, especially when treatment is delayed and/or lymph node suppuration occurs (Rotem et al., 2012).

The aminoglycosides such as gentamicin and streptomycin have a bactericidal activity against $F$. tularensis in vitro, and their use in tularaemia patients is associated with almost $100 \%$ cure rates (Kaya et al., 2011; Rotem et al., 2012). However, they are nephro- and ototoxic and can only be administrated parenterally (Tärnvik and Chu, 2007). Gentamicin is currently used in many countries where streptomycin is no longer available. However, treatment failures with this antibiotic have recently been reported in 11 paediatric patients with oropharyngeal tularaemia in Turkey, with successful recovery after switching to streptomycin (Kaya et al., 2011). Doxycycline can be administrated orally, with few side effects. However, the tetracyclines are contraindicated in 
children under 8 years of age and in pregnant women because of the risk of permanent staining of the dental enamel and bone toxicity in the foetus (Tärnvik and Chu, 2007; Kaya et al., 2011). Treatment with this bacteriostatic antibiotic is associated with higher relapse rates as compared to aminoglycosides and fluoroquinolones, especially when treatment is delayed and/or of short duration (Dennis et al., 2001; Tärnvik and Chu, 2007). Doxycycline is administered for a minimum of 14 days (Dennis et al., 2001; Tärnvik and Chu, 2007). The fluoroquinolones (especially ciprofloxacin and levofloxacin) are preferred as first-line drugs for treatment of tularaemia cases of mild to moderate severity (Johansson et al., 2002). They are bactericidal against $F$. tularensis in vitro, orally administrable, and have few side effects. They can be administrated to young children but not to pregnant women (Johansson et al., 2000; Dennis et al., 2001; Tärnvik and Chu, 2007; Kaya et al., 2011). Ciprofloxacin is recommended as first-line drug in case F. tularensis is used as a biological weapon (Dennis et al., 2001; Rotem et al., 2012).

Other antibiotics such as the beta-lactams, the macrolides, cotrimoxazole, chloramphenicol, and rifampicin are not recommended for treatment of tularaemia. Beta-lactams are not effective both because they are inactivated by the class A betalactamase produced by F. tularensis (Antunes et al., 2012) and they are poorly effective against the intracellular form of this pathogen (Maurin et al., 2000). The macrolides are considered unreliable for treatment of tularaemia because most F. tularensis strains have natural high-level resistance to these antibiotics. Only azithromycin may be a possible alternative in pregnant women infected with type B biovar I strains (Dentan et al., 2013). Chloramphenicol and cotrimoxazole are poorly effective in vitro and potentially associated with severe side effects (Tärnvik and $\mathrm{Chu}, 2007$ ). Rifampicin is active against $F$. tularensis in vitro, but its use as a monotherapy is usually associated with rapid selection of resistant mutants.

No natural strains of $F$. tularensis with acquired resistance to gentamicin, fluoroquinolones or doxycycline have been isolated so far. However, in vitro experiments have shown that mutants resistant to fluoroquinolones, rifampicin or macrolides can be selected easily (Tärnvik and Chu, 2007; Gestin et al., 2010; Sutera et al., 2014). At present, treatment failures and relapses are considered to be primarily related to delayed administration of appropriate antibiotic therapy rather than in vivo selection of antibiotic-resistant mutants (Dennis et al., 2001; Johansson et al., 2002; Kaya et al., 2011; Rotem et al., 2012). However, the bioengineering of genetically modified strains of $F$. tularensis resistant to first-line drugs for use as a biological warfare agent is a major concern. Thus, innovative antibiotics with original structures and bacterial targets, active against this highly virulent pathogen, would be beneficial not only to improve treatment efficacy in tularaemia patients, but also to reinforce our preparedness against the misuse of antibiotic-resistant $F$. tularensis strains.

We recently identified synthetic bis-indole derivatives as new antistaphylococcal compounds with preserved activity against multi-drug resistant strains of Staphylococcus aureus, including MRSA strains (Denis et al., 2013a,b). In this study, we evaluated the activity of four of the leading compounds against clinical isolates of F. tularensis subsp. holarctica.

\section{MATERIALS AND METHODS}

\section{BIS-INDOLIC COMPOUNDS AND ANTIBIOTICS}

The four bis-indolic compounds evaluated in this study $(\mathrm{dcm} 01$, $\mathrm{dcm} 02$, dcm03, and dcm04) were synthesized by the DCM (Département de Chimie Moléculaire, Université Joseph Fourier Grenoble-1, Grenoble, France) according to previously published protocols (Denis et al., 2013a,b). The structures of the tested bis-indole compounds are presented on Figure 1. We also used gentamicin (Panpharma, Fougères, France) and doxycycline (Sigma-Aldrich, Lyon, France) as controls. Stock solutions of the bis-indolic compounds were prepared at $12.8 \mathrm{~g} / \mathrm{L}$ in $100 \%$ DMSO (Sigma-Aldrich, Lyon, France) and stock solutions of gentamicin and doxycycline were prepared in sterile distilled water. All were kept frozen at $-80^{\circ} \mathrm{C}$ until used.

\section{BACTERIAL STRAINS}

All experiments were conducted in a biosafety level 3 laboratory. The use of F. tularensis strains was carried out under the approval of ANSM (Agence nationale de sécurité du médicament et des produits de santé). We tested 41 isolates (Ft1-Ft41) of $F$. tularensis subsp. holarctica identified to the subspecies level in our laboratory (French reference center for Francisella) by amplification and sequencing of the intergenic 16S-23S rRNA region (Maurin et al., 2011). Four isolates were obtained from dead hares and the 37 others from human samples (Table 1). The clinical strains corresponded to independent and sporadic tularaemia cases occurring throughout France between 2004 and 2013 (Maurin et al., 2011). We also tested reference strains including F. tularensis subsp. holarctica LVS NCTC10857, F. tularensis subsp. novicida CIP56.12 and F. philomiragia ATCC25015. S. aureus ATCC29213 was tested as a control strain susceptible to the tested bis-indole compounds (Denis et al., 2013a,b). The reference bacterial strains were purchased from the American Type Culture Collection (ATCC, Mannasas, VA, USA) or the Collection of the Pasteur Institute (CIP, Centre de Ressource Biologique de l'Institut Pasteur, Paris, France). All strains are kept frozen in cryotubes (MastDiagnostic, Amiens, France) at $-80^{\circ} \mathrm{C}$. When needed, they are grown on chocolate agar supplemented

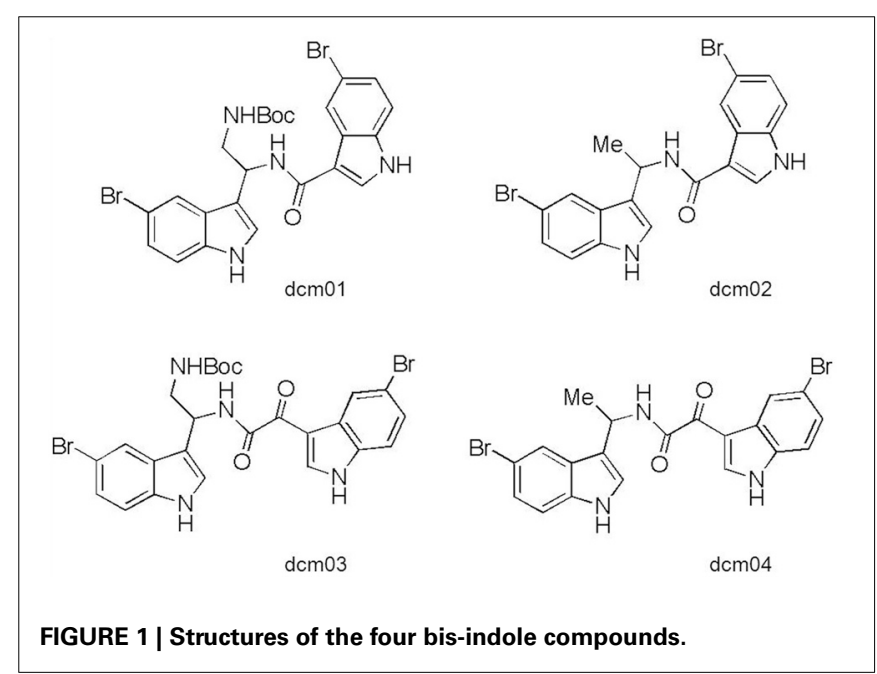


Table 1 | Sources of the 41 isolates of $F$ tularensis subsp. holarctica (Ft1 to $\mathrm{Ft41}$ ) used in this study.

\begin{tabular}{|c|c|c|c|}
\hline Strain & Host & Year of isolation & Clinical sample \\
\hline Ft1 & Animal & UNK & Hare tissue \\
\hline Ft2 & Animal & UNK & Hare tissue \\
\hline Ft3 & Animal & UNK & Hare tissue \\
\hline Ft4 & Animal & UNK & Hare tissue \\
\hline Ft5 & Human & 2004 & Blood culture \\
\hline Ft6 & Human & 2007 & Blood culture \\
\hline Ft7 & Human & 2006 & Conjunctivitis \\
\hline Ft8 & Human & 2007 & Cutaneous ulcer \\
\hline Ft9 & Human & 2007 & Blood culture \\
\hline Ft10 & Human & 2008 & Mediastinal lymph node \\
\hline Ft11 & Human & 2008 & Pharynx \\
\hline Ft12 & Human & 2008 & Pharynx \\
\hline Ft13 & Human & 2008 & Pharynx \\
\hline Ft14 & Human & 2008 & Blood culture \\
\hline Ft15 & Human & 2008 & Cerebrospinal fluid \\
\hline Ft16 & Human & 2008 & UNK \\
\hline Ft17 & Human & 2008 & UNK \\
\hline Ft18 & Human & 2008 & Blood culture \\
\hline Ft19 & Human & 2008 & Blood culture \\
\hline Ft20 & Human & 2008 & Skin ulcer \\
\hline Ft21 & Human & 2008 & Conjunctivitis \\
\hline Ft22 & Human & 2009 & Whitlow \\
\hline Ft23 & Human & 2009 & Middle ear \\
\hline Ft24 & Human & 2009 & Lymph node \\
\hline Ft25 & Human & 2010 & Blood culture \\
\hline Ft26 & Human & 2010 & Blood culture \\
\hline Ft27 & Human & 2010 & Blood culture \\
\hline Ft28 & Human & 2010 & Lymph node \\
\hline Ft29 & Human & 2010 & Blood culture \\
\hline Ft30 & Human & 2011 & Lymph node \\
\hline Ft31 & Human & 2011 & UNK \\
\hline Ft32 & Human & 2012 & UNK \\
\hline Ft33 & Human & 2011 & Blood culture \\
\hline Ft34 & Human & 2012 & Blood culture \\
\hline Ft35 & Human & 2012 & Finger abscess \\
\hline Ft36 & Human & 2012 & UNK \\
\hline Ft37 & Human & 2010 & Blood culture \\
\hline Ft38 & Human & 2012 & Blood culture \\
\hline Ft39 & Human & 2012 & Whitlow \\
\hline $\mathrm{Ft} 40$ & Human & 2012 & Pleural fluid \\
\hline $\mathrm{Ft} 41$ & Human & 2013 & Pleural fluid \\
\hline
\end{tabular}

UNK, unknown.

with Polyvitex ${ }^{\circledR}$ (CHA-PVX medium, bioMérieux, Marcy l'Etoile, France) at $37^{\circ} \mathrm{C}$ in a $5 \% \mathrm{CO}_{2}$-enriched atmosphere.

\section{DETERMINATION OF THE MINIMUM INHIBITORY CONCENTRATIONS}

Minimum inhibitory concentrations (MICs) of the four bis-indolic compounds were determined against $S$. aureus ATCC29213, F. philomiragia ATCC25015 and all F. tularensis strains, using a broth microdilution method recommended by the Clinical and Laboratory Standards Institute (CLSI;
M07-A8 Vol. 29, No. 2). Mueller-Hinton 2 broth supplemented with 2\% PolyViteX ${ }^{\circledR}$ (MH2-PVX, bioMérieux, Marcy L'Etoile, France) was used as the antibiotic susceptibility testing medium for $F$. tularensis strains because of their fastidious nature. $\mathrm{MH} 2$ alone was used for other species. One row of a 96-well microtiter plate was filled with $75 \mu \mathrm{L}$ of twofold serial dilutions of the tested bis-indolic compound in MH2-PVX medium, so as to obtain final bis-indolic concentrations ranging from 0.06 to $32 \mu \mathrm{g} / \mathrm{mL}$ in $0.5 \%$ DMSO. A bacterial inoculum $(75 \mu \mathrm{L}$ per well, $5 \times 10^{5} \mathrm{CFU} / \mathrm{mL}$ of final inoculum) was then added to each well. Antibiotic free cultures containing 0.5\% DMSO were used as DMSO toxicity controls. MH2-PVX medium with $0.5 \%$ DMSO served as a negative control. Microplates were incubated at $37^{\circ} \mathrm{C}$ in a $5 \% \mathrm{CO}_{2}$ atmosphere. The MICs were read after $18 \mathrm{~h}$ culture incubation for S. aureus ATCC29213 and $48 \mathrm{~h}$ for F. tularensis strains. MICs corresponded to the minimum bis-indolic compound concentration that allowed complete inhibition of visual growth of bacteria. Experiments were conducted at least twice to confirm results. Following the same procedure but without DMSO, the MICs of gentamicin and doxycycline were determined against F. tularensis subsp. holarctica Ft6 and Ft24 strains and the control strain S. aureus ATCC29213.

\section{DETERMINATION OF THE MINIMUM BACTERICIDAL CONCENTRATIONS}

Minimum bactericidal concentrations (MBCs) were determined in triplicate experiments following CLSI recommendations (CLSI, M26-A, Vol. 19, No. 18), for the two most active compounds (dcm02 and dcm04) against three F. tularensis subsp. holarctica strains: the LVS strain and the two clinical strains Ft6 and Ft24. We used the same microdilution broth method described for MIC determination, but the primary bacterial inoculum was $10^{6} \mathrm{CFU} / \mathrm{mL}$. MBCs of gentamicin and doxycycline were determined in parallel as a bactericidal and a bacteriostatic control, respectively. After $48 \mathrm{~h}$ incubation at $37^{\circ} \mathrm{C}$ in a $5 \%$ $\mathrm{CO}_{2}$ atmosphere, MBCs were determined by plating $50 \mu \mathrm{L}$ of tenfold serial dilutions of the bacterial suspensions of wells with no visible growth, and of the antibiotic-free control well, onto CHAPVX medium. CFU counts were determined after $72 \mathrm{~h}$ incubation of the plates at $37^{\circ} \mathrm{C}$, in a $5 \% \mathrm{CO}_{2}$ atmosphere. The detection limit was $20 \mathrm{CFU} / \mathrm{mL}$. The $\mathrm{MBC}$ corresponded to the minimal antibiotic concentration which resulted in at least $99.9 \%$ reduction of the primary bacterial inoculum (i.e., $3 \log _{10}$ reduction of bacterial titers).

\section{TIME-KILL CURVES}

Time-kill curves were determined for the LVS, Ft6, and Ft24 strains and the leading dcm04 compound. The primary inoculum calibrated at $10^{6} \mathrm{CFU} / \mathrm{mL}$ was prepared in MH2-PVX medium and split into five $5-\mathrm{mL}$ aliquots: one drug-free control; three others receiving 4, 8, and 16 times, respectively, the MIC of dcm04 for the tested strain, with $0.5 \%$ final concentration of DMSO in all three aliquots; and the last one receiving eight times the MIC of gentamicin for the tested strain, used as a positive control. Sterile $\mathrm{MH} 2-\mathrm{PVX}$ medium with $0.5 \% \mathrm{DMSO}$ served as a negative control. Cultures were incubated $48 \mathrm{~h}$ at $37^{\circ} \mathrm{C}$ in $5 \% \mathrm{CO}_{2}$. At $0,6,12$, 24,36 , and $48 \mathrm{~h}$ of incubation, a $50-\mu \mathrm{L}$ aliquot was taken from 
Table 2 | MICs $(\mu \mathrm{g} / \mathrm{mL})$ of Francisella strains for the four bis-indolic compounds: dcm01, dcm02, dcm03, and dcm04.

\begin{tabular}{|c|c|c|c|c|c|c|}
\hline \multirow[t]{3}{*}{ Bis-indole compound } & \multicolumn{4}{|c|}{ F tularensis subsp. holarctica } & \multirow{3}{*}{$\begin{array}{l}\text { subsp. novicida } \\
\text { CIP } 56.12 \\
\text { MIC }\end{array}$} & \multirow{3}{*}{$\begin{array}{c}\text { F philomiragia } \\
\text { ATCC25015 } \\
\text { MIC }\end{array}$} \\
\hline & \multicolumn{3}{|c|}{ FT1 to Ft41 } & \multirow{2}{*}{$\begin{array}{l}\text { LVS } \\
\text { MIC }\end{array}$} & & \\
\hline & MIC range & $\mathrm{MIC}_{50}$ & $\mathrm{MIC}_{90}$ & & & \\
\hline $\mathrm{dcm} 01$ & $2->32$ & 4 & 8 & 2 & $>32$ & $>32$ \\
\hline dcm02 & $4-8$ & 4 & 8 & 2 & 32 & 16 \\
\hline
\end{tabular}

each culture after shaking. Then $50 \mu \mathrm{L}$ of ten-fold serial dilutions of each aliquot was plated on CHA-PVX medium. CFU counts were determined after $72 \mathrm{~h}$ incubation of the plates at $37^{\circ} \mathrm{C}$ in $5 \% \mathrm{CO}_{2}$. The detection limit was $20 \mathrm{CFU} / \mathrm{mL}$. A $3-\log _{10}$ or more reduction of the primary bacterial inoculum at any incubation time was considered a significant bactericidal effect. Experiments were conducted at least twice to confirm the results.

\section{STATISTICAL ANALYSIS}

A statistically significant decrease of viable bacterial counts in MBC assays was evaluated by one-tailed Student t-test using Statview ${ }^{\circledR}$ software. For each antibiotic concentration tested, we compared the bacterial count obtained after $48 \mathrm{~h}$ of incubation of cultures to the primary inoculum [i.e., $\log (\mathrm{N} / \mathrm{N} 0)]$ and to a 3-log reduction cutoff. Significance was defined as a $p$-value $<0.05$.

\section{RESULTS}

\section{ALL TESTED SYNTHETIC BIS-INDOLIC COMPOUNDS ARE ACTIVE AGAINST F. TULARENSIS SUBSP. HOLARCTICA BUT NOT F. TULARENSIS SUBSP. NOVICIDA}

MICs are represented in Tables 2, 3. Almost all F. tularensis strains tested were susceptible to the four bis-indole derivatives. In contrast, the Ft5 strain was susceptible to $\mathrm{dcm} 02$ and $\mathrm{dcm} 04$ ( $\mathrm{MIC}=$ 8 and $2 \mu \mathrm{g} / \mathrm{mL}$, respectively), but resistant to $\mathrm{dcm} 01$ and $\mathrm{dcm} 03$ (MICs $>32 \mu \mathrm{g} / \mathrm{mL}$ ). Dcm04 was the most active bis-indole compound with MICs ranging from 2 to $4 \mu \mathrm{g} / \mathrm{mL}$ and a $\mathrm{MIC}_{90}$ of $2 \mu \mathrm{g} / \mathrm{mL}$. The $\mathrm{MIC}_{90}$ of the three other compounds was $8 \mathrm{mg} / \mathrm{L}$. It should be noted that prolonged incubation of cultures only increased MICs by one dilution for some compounds. In comparison, the MIC of gentamicin against the LVS, Ft6 and Ft24 strains was $0.25 \mu \mathrm{g} / \mathrm{mL}$ and the MIC of doxycycline was $0.125 \mu \mathrm{g} / \mathrm{mL}$ against LVS and $0.25 \mu \mathrm{g} / \mathrm{mL}$ against the Ft6 and Ft24 strains. As for control strains, the F. tularensis subsp. holartica LVS strain was susceptible to the four bis-indole derivatives with a MIC of $1-2 \mu \mathrm{g} / \mathrm{mL}$. In contrast, the reference $F$. tularensis subsp. novicida strain and the reference $F$. philomiragia strain were more resistant to these compounds. MICs of all bis-indole derivatives were $\geq 32 \mu \mathrm{g} / \mathrm{mL}$ for F. tularensis subsp. novicida, whereas $\mathrm{dcm} 02$ and dcm 04 displayed lower MICs ( 16 and $4 \mu \mathrm{g} / \mathrm{mL}$, respectively) against F. philomiragia.

\section{MBC DETERMINATION AND TIME-KILL STUDIES REVEALED A BACTERICIDAL ACTIVITY AGAINST THE LVS STRAIN BUT ONLY BACTERIOSTATIC ACTIVITY AGAINST THE Ft6 AND Ft24 STRAINS}

The bactericidal activities of the bis-indole compounds, gentamicin and doxycycline, were determined for the LVS, Ft6, and Ft24
Table 3 | MICs $(\mu \mathrm{g} / \mathrm{mL})$ and MBCs $(\mu \mathrm{g} / \mathrm{mL})$ of the tree $F$ tularensis subsp. holarctica strains used for time-kill studies and MBC determination.

\begin{tabular}{lcccc}
\hline $\begin{array}{l}\text { Bacterial } \\
\text { strain }\end{array}$ & \multicolumn{4}{c}{ MIC (MBC) } \\
\cline { 2 - 5 } & $\mathbf{d c m 0 2}$ & $\mathbf{d c m 0 4}$ & Gentamicin & Doxycycline \\
\hline $\begin{array}{l}\text { F. tularensis } \\
\text { subsp. } \\
\text { holarctica Ft6 }\end{array}$ & 4 & 2 & $0.25(2)$ & 0.25 \\
$\begin{array}{l}\text { F. tularensis } \\
\text { subsp. } \\
\text { holarctica Ft24 }\end{array}$ & 4 & 2 & $0.25(2)$ & 0.25 \\
$\begin{array}{l}\text { F. tularensis } \\
\text { subsp. } \\
\text { holarctica LVS }\end{array}$ & $2(4)$ & $1(4)$ & $0.25(2)$ & 0.125 \\
\hline
\end{tabular}

strains (Figures 2, 3). As expected, gentamicin displayed bactericidal activity against the three strains $\left(>3 \log _{10}\right.$ reduction of the primary bacterial inoculum, $p<0.01$ ), with MBCs of $1 \mu \mathrm{g} / \mathrm{mL}$ for the LVS strain and $2 \mu \mathrm{g} / \mathrm{mL}$ for Ft6 and Ft24 strains. As for doxycycline, a significant reduction of the primary bacterial inoculum was observed (i.e., between 1 and $2 \log _{10}$ at MIC $\times 64$ for the LVS strain and MIC $\times 32$ for the Ft 6 and Ft24 strains; $p<0.01$ ) but the 3 -log reduction cutoff was not reached.

MBCs of the dcm02 and dcm04 compounds $(4 \mu \mathrm{g} / \mathrm{mL}$ for both compounds) were only two or four times their respective MICs for the LVS strain (Table 3), respectively. In contrast, MBCs could not be determined for the $\mathrm{dcm} 02$ and $\mathrm{dcm} 04$ compounds against the Ft6 and Ft24 strains. Here again reduction of the primary bacterial inoculum was significant (2-log reduction at concentrations up to MIC $\times 8$ for $\mathrm{dcm} 02$ and MIC $\times 16$ for dcm04, $p<$ 0.01 ) but did not reached the 3 -log cutoff. Higher concentrations of these compounds could not be tested because of their poor solubility.

Time-kill studies revealed a $3-\log _{10}$ reduction of the primary inoculum of the LVS strain after $12 \mathrm{~h}$ incubation for gentamicin $(\mathrm{MIC} \times 8)$ and $24 \mathrm{~h}$ for $\mathrm{dcm} 04(\mathrm{MIC} \times 4)$. As for $\mathrm{dcm} 04$, the same bactericidal kinetics were observed at 4,8 , and 16 times the MIC of this compound for the LVS strain. As for the Ft6 and Ft24 strains, we observed a progressive decrease of the bacterial load over the first $48 \mathrm{~h}$ of contact with dcm04 (Figure 3), but a 3- $\log _{10}$ reduction of the primary bacterial inoculum was never reached. Thus, the dcm02 and dcm04 compounds were only bacteriostatic against the clinical strains of $F$. tularensis subsp. holarctica. 

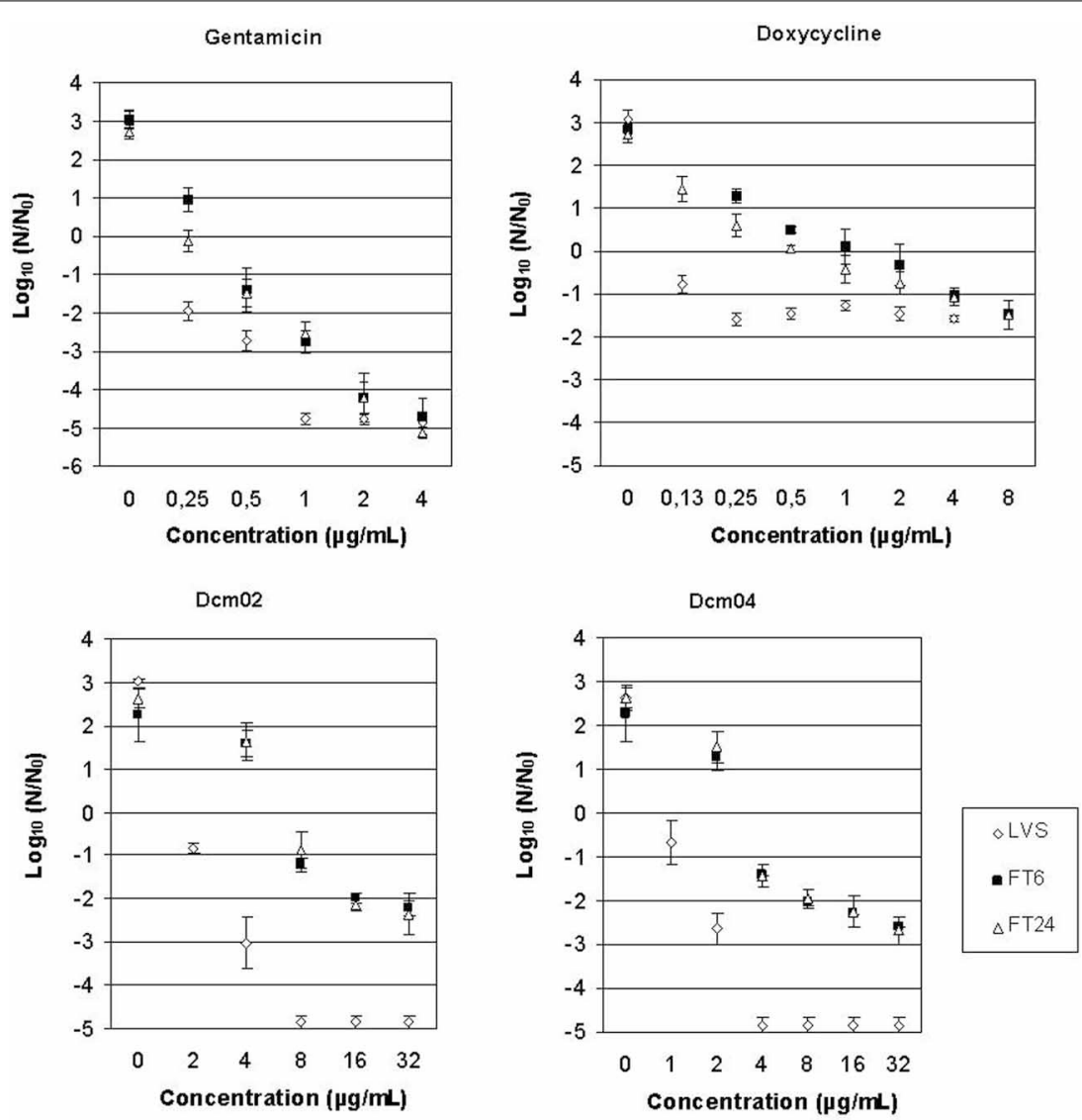

FIGURE 2 | MBC determination figures for dcm02, dcm04, gentamicin, and doxycycline against $F$. tularensis subsp. holarctica LVS, Ft6, and Ft24 strains, performed in triplicate experiments. Bacterial survival was monitored by measuring $\mathrm{CFU} / \mathrm{mL}$ after $48 \mathrm{~h}$ of incubation. Error bars represent standard deviation.

\section{DISCUSSION}

Among the currently developed therapeutic alternatives for tularaemia, two promising original classes of compounds have recently been identified. First, substituted diphenyl ethers have demonstrated potent inhibition of ftuFabI Enoyl-acyl carrier protein reductase (England et al., 2009). This enzyme, absent in human cells, plays a key role in the type II fatty acid biosynthesis and has proved to be a useful target for growth inhibition of various pathogens such as Mycobacterium tuberculosis, S. aureus and Plasmodium falciparum (England et al., 2009; Lu et al., 2009; Hevener et al., 2012; Mehboob et al., 2012; Kingry et al., 2013). The leading compound SBPT04 has an MIC of $0.16 \pm$ $0.06 \mu \mathrm{g} / \mathrm{mL}$ against F. tularensis LVS and Schu4 strains and also has a bactericidal activity with a MBC of $0.25 \mu \mathrm{g} / \mathrm{mL}$. In a murine model of $F$. tularensis infection, this compound cleared bacteria by day 4 of treatment, without any relapse the following 30 days post-treatment (England et al., 2009). Secondly, screening of a library of more than 1000 2,5,6- and 2,5,7-trisubstituted benzimidazoles identified 21 leading derivatives exhibiting MICs between 0.35 and $48.6 \mu \mathrm{g} / \mathrm{mL}$ against the F. tularensis LVS strain. Their bacterial target remains uncharacterized, but these compounds may block polymerization of FtsZ, which is a homolog of tubulin/microtubule proteins found in eukaryotes, thus interfering with cell division processes (Kumar et al., 2013).
Here, we report that bis-indole derivatives in which the two indole groups are linked either with an amide (dcm01 and $\mathrm{dcm} 02)$ or an $\alpha$-keto-amide $(\mathrm{dcm} 03$ and $\mathrm{dcm} 04)$ central linker exhibit antimicrobial activity against $F$. tularensis subsp. holarctica. These compounds were previously characterized as anti-staphylococcal drugs active against methicillin-resistant, vancomycin-intermediate, and fluoroquinolone-resistant $S$. aureus strains (Denis et al., 2013a,b). In this study, the 24 bisindolic molecules evaluated were inactive against Gram-negative bacteria, including enterobacterial species (Escherichia coli, Klebsiella pneumoniae, Serratia marcescens, and Enterobacter cloacae), Pseudomonas aeruginosa and Acinetobacter baumannii. The MICs of two bis-indolic derivatives were lower $(16 \mu \mathrm{g} / \mathrm{mL})$ against Haemophilus influenzae. The cytotoxicity of the four bis-indole compounds we tested against F. tularensis strains was previously evaluated using three different cell lines: KB (human mouth carcinoma), MCR5 (human lung fibroblast) and HCT116 (human colon tumor) (Denis et al., 2013a,b). The $\mathrm{IC}_{50}$ determined using the HCT116 cell line were 1-5 times higher than the MICs found for F. tularensis strains.

We found a significant bacteriostatic activity of these tested bis-indole derivatives against 41 strains of F. tularensis subsp. holarctica isolated in France. Dcm04 appeared to be the most 
FT6

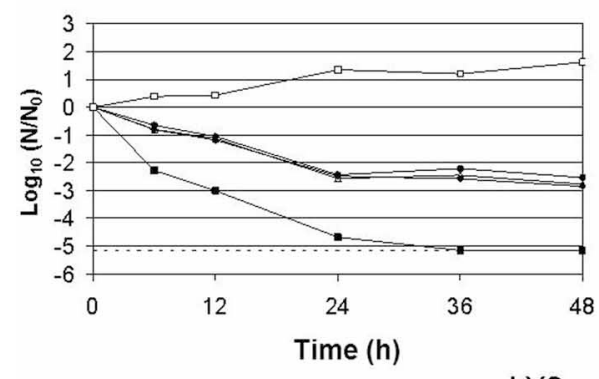

LVS
FT24

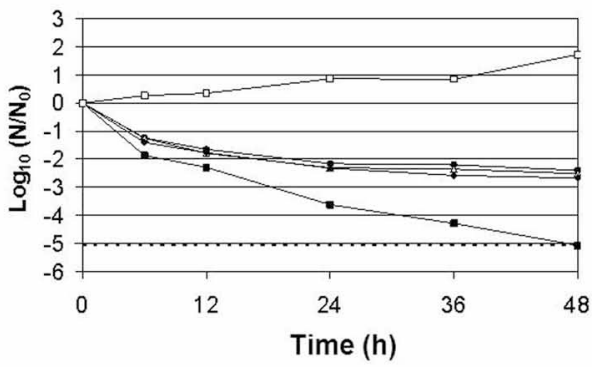

Time (h)
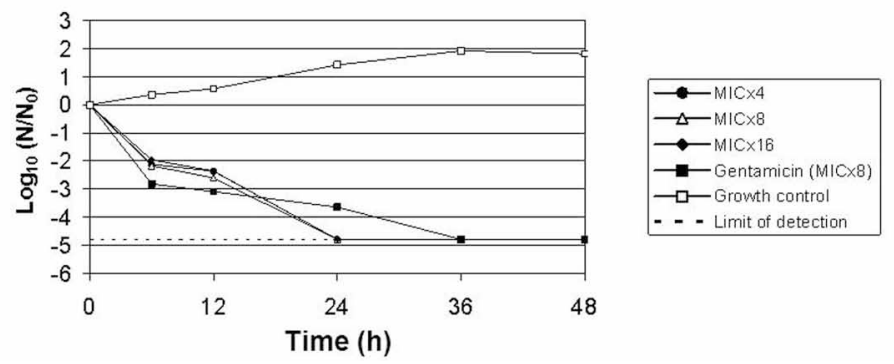

FIGURE 3 | Time-kill kinetics of dcm04 at 4, 8, and 16 times the MIC against $F$ tularensis subsp. holarctica LVS, Ft6, and Ft24 strains. Gentamicin at eight times the MIC was used as control of a bactericidal antibiotic. Two independent assays were performed but only one representative experiment is shown.

effective compound with a $\mathrm{MIC}_{90}$ of $2 \mu \mathrm{g} / \mathrm{mL}$. The MICs ranged from 2 to $16 \mu \mathrm{g} / \mathrm{mL}$ when considering all four bis-indole compounds, except for a single strain that displayed higher MICs for the dcm01 and dcm03 compounds. The variations in antibiotic activities between the four compounds (especially between $\mathrm{dcm} 01$ and $\mathrm{dcm} 03$ vs. $\mathrm{dcm} 02$ and $\mathrm{dcm} 04$ ) may be related to differences in chemical structure, especially the presence of a large $\mathrm{CH}_{2} \mathrm{NHBoc}$ chemical group in $\mathrm{dcm} 01$ and $\mathrm{dcm} 03$, whereas it is replaced by a methyl in dcm02 and dcm04. This large chemical group may limit access of $\mathrm{dcm} 01$ and $\mathrm{dcm} 03$ to their bacterial target or limit their penetration within bacteria. Surprisingly, the $\mathrm{dcm} 02$ and $\mathrm{dcm} 04$ compounds displayed a bactericidal activity against the virulence-attenuated LVS strain, but not the Ft6 and Ft24 clinical strains of $F$. tularensis subsp. holarctica. This was demonstrated both by MBC determinations and in killing curve experiments. The bactericidal activity of dcm04 against the LVS strain was not concentration-dependent but time-dependent. Hopefully, further structural optimization of these bis-indolic compounds and identification of their bacterial targets may enable us to obtain the same bactericidal activity for clinical strains of F. tularensis subsp. holarctica.

The activity of the bis-indole compounds also showed Francisella species and subspecies specificity, since these compounds were active against $F$. tularensis subsp. holarctica, only partially active $(\mathrm{dcm} 02$ and $\mathrm{dcm} 04)$ against $F$. philomiragia and inactive against $F$. tularensis subsp. novicida. As a result, F. tularensis subsp. novicida cannot be used as an experimental model to identify the bacterial targets of these compounds, nor to evaluate the in vivo activity of the bis-indoles. Comparison of the complete genomes of $F$. tularensis subsp. holarctica LVS, OSU18, and FSC200, and that of F. tularensis subsp. tularensis Schu
S4, previously identified a relatively limited number of specific genetic alterations (Petrosino et al., 2006; Rohmer et al., 2006) in the attenuated LVS strain. This might help in further investigations to search for an antibacterial target of these compounds in F. tularensis, or at least to explain the differences observed in susceptibility to the bis-indoles. Working hypotheses may include an increased affinity of the bis-indoles for their bacterial target in the LVS strain, a reduced penetration of the bis-indole in the clinical strains as compared to the LVS strain, partial inactivation of the bis-indoles in the clinical strains but not in the LVS strain, an escape pathway to the action of the bis-indoles in clinical strains but not in the LVS strain, and a lower efflux of these molecules in the LVS strain. Another hypothesis is that the bisindole compounds are more active against the LVS strain because it belongs to the type B biovar II strains of F. tularensis, whereas the 41 clinical strains belong to biovar I of this sub-species. Biovar II strains naturally resistant to erythromycin are found in Central and Eastern Europe, and Asia (Kudelina and Olsufiev, 1980), whereas only the erythromycin-susceptible biovar I strains are found in France. We did not evaluate the activity of the bisindoles against type A F. tularensis strains. Testing the activity of these compounds against a larger panel of F. tularensis strains will be needed to assess potential variability in susceptibility among different sub-species and biovars.

In conclusion, we have identified novel synthetic bis-indole compounds active against F. tularensis subsp. holarctica but not the closely related bacteria $F$. tularensis subsp. novicida and F. philomiragia. These compounds may be drug candidates for the development of new therapeutic alternatives for tularaemia treatment. Their bacterial targets remain to be characterized. 


\section{AUTHOR CONTRIBUTIONS}

Research project design: Yvan Caspar, Max Maurin. Experiments: Yvan Caspar, Vivien Sutera, Sandrine Boisset. Writing: Yvan Caspar, Max Maurin.

\section{ACKNOWLEDGMENTS}

The authors thank Linda Northrup for English editing. This work was supported by the Institut National de Veille Sanitaire (InVS), the Direction Générale de l'Armement (DGA, 07CO301), and the Centre Hospitalo-Universitaire (CHU) of Grenoble, Joseph Fourier University-Grenoble 1.

\section{REFERENCES}

Antunes, N. T., Frase, H., Toth, M., and Vakulenko, S. B. (2012). The class A $\beta$-Lactamase FTU-1 is native to Francisella tularensis. Antimicrob. Agents Chemother. 56, 666-671. doi: 10.1128/AAC.05305-11

Denis, J.-N., Jolivalt, C., Maurin, M., and Burchak, O. (2013a). Preparation of bisindole derivatives useful as antibacterials. PCT Int. Appl. WO $2013014104 \mathrm{Al}$ 20130131.

Denis, J.-N., Jolivalt, C., Maurin, M. and Jeanty, M. (2013b). Preparation of novel bis-indolic derivatives antibacterial drugs and a process for preparing them. PCT Int. Appl. WO 2013014102 A1 20130131.

Dennis, D. T., Inglesby, T. V., Henderson, D. A., Bartlett, J. G., Ascher, M. S., Eitzen, E., et al. (2001). Tularemia as a biological weapon: medical and public health management. JAMA 285, 2763-2773. doi: 10.1001/jama.285.21.2763

Dentan, C., Pavese, P., Pelloux, I., Boisset, S., Brion, J.-P., Stahl, J.-P., et al. (2013). Treatment of tularemia in pregnant woman, France. Emerging Infect. Dis. 19, 996-998. doi: 10.3201/eid1906.130138

England, K., am Ende, C., Lu, H., Sullivan, T. J., Marlenee, N. L., Bowen, R. A., et al. (2009). Substituted diphenyl ethers as a broad-spectrum platform for the development of chemotherapeutics for the treatment of tularaemia. J. Antimicrob. Chemother. 64, 1052-1061. doi: 10.1093/jac/dkp307

Gestin, B., Valade, E., Thibault, F., Schneider, D., and Maurin, M. (2010). Phenotypic and Genetic characterization of macrolide resistance in Francisella tularensis subsp. holarctica biovar I. J. Antimicrob. Chemother. 65, 2359-2367. doi: $10.1093 / \mathrm{jac} / \mathrm{dkq} 315$

Hevener, K. E., Mehboob, S., Su, P.-C., Truong, K., Boci, T., Deng, J., et al. (2012). Discovery of a novel and potent class of F. tularensis enoyl-reductase (FabI) inhibitors by molecular shape and electrostatic matching. J. Med. Chem. 55, 268-279. doi: 10.1021/jm201168g

Johansson, A., Berglund, L.,Gothefors, L., Sjöstedt, A., and Tärnvik. A. (2000). Ciprofloxacin for treatment of tularemia in children. Pediatr. Infect. Dis. J. 19, 449-453. doi: 10.1097/00006454-200005000-00011

Johansson, A., Urich, S.K., Chu, M. C., Sjöstedt, A., and Tärnvik, A. (2002). In vitro susceptibility to quinolones of Francisella tularensis subsp. tularensis. Scand. J. Infect. Dis. 34, 327-330. doi: 10.1080/00365540110080773

Kaya, A., Uysal, I. O., Güven, A. S., Engin, A., Gültürk, A., Íçağası oğlu, F. D., et al. (2011). Treatment failure of gentamicin in pediatric patients with oropharyngeal tularemia. Med. Sci. Monit. 17, 376-380. doi: 10.12659/MSM.881848

Kingry, L. C., Cummings, J. E., Brookman, K. W., Bommineni, G. R., Tonge, P. J., and Slayden, R. A. (2013). The Francisella tularensis FabI enoyl-acyl carrier protein reductase gene is essential to bacterial viability and is expressed during infection. J. Bacteriol. 195, 351-358. doi: 10.1128/JB.01957-12

Kudelina, R. I., and Olsufiev, N. G. (1980). Sensitivity to macrolide antibiotics and lincomycin in Francisella tularensis holarctica. J. Hyg. Epidemiol. Microbiol. Immunol. 24, 84-91.

Kumar, K., Awasthi, D., Lee, S.-Y., Cummings, J. E., Knudson, S. E., Slayden, R. A., et al. (2013). Benzimidazole-based antibacterial agents against Francisella tularensis. Bioorg. Med. Chem. 21, 3318-3326. doi: 10.1016/j.bmc.2013.02.059
Larssen, K. W., Afset, J. E., Heier, B. T., Krogh, T., Handeland, K., Vikøren, T., et al. (2011). Outbreak of tularaemia in central norway, January to March 2011. Euro Surveill. 16:pii 19828. Available online at: http://www.eurosurveillance.org/ViewArticle.aspx?ArticleId=19828

Lu, H., England, K., am Ende, C., Truglio, J. J., Luckner, S., Reddy, B. G., et al. (2009). Slow-onset inhibition of the FabI enoyl reductase from Francisella tularensis: residence time and in vivo activity. ACS Chem. Biol. 4, 221-231. doi: $10.1021 / \mathrm{cb} 800306 y$

Maurin, M., Mersali, N. F., and Raoult, D. (2000). Bactericidal activities of antibiotics against intracellular Francisella tularensis. Antimicrob. Agents Chemother. 44, 3428-3431. doi: 10.1128/AAC.44.12.3428-3431.2000

Maurin, M., Pelloux, I., Brion, J.-P., Del Banõ, J.-N. and Picard, A. (2011). Human tularemia in France, 2006-2010. Clin. Infect. Dis. 53, e133-e141. doi $10.1093 / \mathrm{cid} / \mathrm{cir} 612$

Mehboob, S., Hevener, K. E., Truong, K., Boci, T., Santarsiero, B. D., and Johnson, M. E. (2012). Structural and enzymatic analyses reveal the binding mode of a novel series of Francisella tularensis enoyl reductase (FabI) inhibitors. J. Med. Chem. 55, 5933-5941. doi: 10.1021/jm300489v

Pérez-Castrillón, J. L., Bachiller-Luque, P., Martin-Luquero, M., Mena-Martin, F. J., and Herreros, V. (2001). Tularemia epidemic in northwestern Spain: clinical description and therapeutic response. Clin. Infect. Dis. 33, 573-576. doi: $10.1086 / 322601$

Petrosino, J. F., Xiang, Q., Karpathy, S. E., Jiang, H., Yerrapragada, S., Liu, Y., et al. (2006). Chromosome rearrangement and diversification of Francisella tularensis revealed by the type B (OSU18) genome sequence. J. Bacteriol. 188, 6977-6985. doi: 10.1128/JB.00506-06

Rohmer, L., Brittnacher, M., Svensson, K., Buckley, D., Haugen, E., Zhou, Y., et al. (2006). Potential source of Francisella tularensis live vaccine strain attenuation determined by genome comparison. Infect. Immun. 74, 6895-6906. doi: 10.1128/IAI.01006-06

Rotem, S., Bar-Haim, E., Cohen, H., Elia, U., Ber, R., Shafferman, A., et al. (2012). Consequences of delayed ciprofloxacin and doxycycline treatment regimens against Francisella tularensis airway infection. Antimicrob. Agents Chemother. 56, 5406-5408. doi: 10.1128/AAC.01104-12

Rydén, P., Björk, R., Schäfer, M. L., Lundström, J. O., Petersén, B., Lindblom, A., et al. (2012). Outbreaks of tularemia in a boreal forest region depends on mosquito prevalence. J. Infect. Dis. 205, 297-304. doi: 10.1093/ infdis/jir732

Sutera, V., Levert, M., Burmeister, W. P., Schneider, D., and Maurin, M. (2014). Evolution toward high-level fluoroquinolone resistance in Francisella species. J. Antimicrob. Chemother. 69, 101-110. doi: 10.1093/jac/dkt321

Tärnvik, A., and Chu, M. C. (2007). New approaches to diagnosis and therapy of tularemia. Ann. N.Y Acad. Sci. 1105, 378-404. doi: 10.1196/annals.1409.017

Conflict of Interest Statement: The authors declare that the research was conducted in the absence of any commercial or financial relationships that could be construed as a potential conflict of interest.

Received: 13 January 2014; accepted: 12 February 2014; published online: 27 February 2014.

Citation: Caspar Y, Sutera V, Boisset S, Denis J-N and Maurin M (2014) Bis-indolic compounds as potential new therapeutic alternatives for tularaemia. Front. Cell. Infect. Microbiol. 4:24. doi: 10.3389/fcimb.2014.00024

This article was submitted to the journal Frontiers in Cellular and Infection Microbiology.

Copyright (c) 2014 Caspar, Sutera, Boisset, Denis and Maurin. This is an openaccess article distributed under the terms of the Creative Commons Attribution License (CC BY). The use, distribution or reproduction in other forums is permitted, provided the original author(s) or licensor are credited and that the original publication in this journal is cited, in accordance with accepted academic practice. No use, distribution or reproduction is permitted which does not comply with these terms. 